\title{
A New Game Console: Immerse Helmet
}

\author{
Boyang Yu, Mochen Xia, Yue Zhang \\ Beijing University of Posts and Telecommunications, Beijing, China
}

Keywords: New game console, immersive 3D, OLED, APU

\begin{abstract}
Recently, the game console industry has been developing at a considerable rate. Also, the development of 3D technology is fast and promising. In the present market, there are already game consoles and games providing users with 3D perception. But actually, the technology they used is called non-immersive 3D, users can only experience the 3D view in a fixed direction from their eyesight, while the side of the scene cannot be seen. So we designed a kind of game console combining the new processing chip, new OLED material, and the immersive 3D technology in order to improve the immersion of the current game devices. It will have better processing speed, and will break the width limitation of the scene.
\end{abstract}

\section{INTRODUCTION}

\subsection{The Development of Game Console}

In 1962, the Massachusetts Institute of Technology student Steve Russell and several of his classmates designed a PC game -- 'Space War'. The game used the cathode ray tube display to reveal images and simulates a space which contains various planets. In this space, physical properties such as gravity, acceleration, inertial and so on are all considered. Game players use a pair of special controllers to control a variety of weapons to destroy each other's space ship and to avoid colliding the planet. SpaceWar is the first video game for entertainment, it came out 4 year later than the world's first electronic game 'Tennis for Two'.

Then in 1972, Maganavox's new device 'Odyssey' was designed by Ralph Baer. It is the first console for family game.

PC Engine came out in 1987. It was extremely popular in Japan. It can issue 512 colors in different resolution at the same time, and has a perfect ability of image mosaic. The chroma coder which is designed by Hudson can transmit more vivid and brighter color image signals. PC Engine is the first console for family use with the optional CD module, which has the benefits of the CD carrier (more storage space, cheaper carrier cost, can play Red Book standard CD music, etc.).

Two years later, in 1989, GameBoy appeared on the market. It used a four-color black and white screen and the CPU is just 8-bits. At the same time the maximum capacity of the game card is just 32MB.

3DO Company and Panasonic cooperated the first 32-bits host machine -- 3DO in 1994, which the screen resolution is just $640 \times 480$ at that time.

Then in 1996, the Nintendo developed the third generation home console N64, the follow-up model of SFC. Its biggest innovation is that it added analog joysticks and handle vibration bag on the handle for the first time. Two years later, Nintendo developed Game Boy Color (GBC) which used battery driven and color screen in 1998, and it supported a maximum of 32,000 colors.

In 2001, Nintendo sold the second generation portable game machine GBA. The biggest feature of GBA is the use of the TFT liquid crystal screen. GBA can be compatible with Game Boy and Game Boy Color games. In the same year, Gamepark Corporation developed the handheld game machine GP32. Its host stressed the side effect of miracle and used a 3.5 inch TFT-LCD, which improved the disadvantage of dark picture in STN-LCD, and also solved the problem of picture afterimage to make the action games flash more fluently in GP32.

GBAsp, the enhanced console of GBA came out in 2003. The kernel is identical to GBA, but used a different appearance and screen. GBAsp can be folded so the fragile screen can be protected, 
and it also added the background light so that users can also enjoy the entertainment pleasure in dark night. Besides, GBAsp also added the rechargeable battery, which can ensure the power supply of it.

In 2004, NDS came out, its wireless network will provide a 10 to 30 meters communication distance, and users can use the built-in microphone to communicate through the internet. It contained six languages (Japanese, English, German, French, Spanish and Italian), and can record the input status information of the user. NDS used a rechargeable lithium ion battery, which can work 4 to 10 hours (for software varies). It takes 4 hours for fully charged, as well as the sleep mode and other related power management functions are provided to prolong the use of the time.

Then in 2006, Wii was launched by Nintendo, which lead the game device into a new field of somatosensory game. The machine for somatosensory game is based on i-wall and i-hockey standard, which rely on high-tech video motion capture technology that can reflect the physical action immediately into the game system such as Xbox 360 and Sony PS3, which give users a better game experience.

\subsection{The Development of 3D Technology}

The development of 3D technology can be traced back to 280 B.C.E. Euclid of Greece recognized that simultaneously looking at slightly different scenes of the same object, as viewed from the left and right, produces a 3D effect. In 1600, Giovanni Battista della Porta of Italy tried to create a 3D image by arranging pictures of objects viewed from the left and right. In 1838, Sir Charles Wheatstone of England demonstrated a 3D display using mirrors to combine stereoscopic images.

In 1844, invented by a Scottish physicist David Brewster, Stereoscope drew people's attention. It is the first equipment used for creating three-dimension view in the photography field. Soon after, at the Great Exhibition in 1851, a picture of Queen Victoria made the technology well known all over the world.

In the late 19th century, after the invention of cinema, scientists tried to demonstrate the movement of the stereoscopic images. In 1915, the first anaglyph movie appeared. Immediately after, "The Power of Love", the first published 3D movie was produced in 1922, and in 1935, the first 3D color movie was brought to the public view. 3D technology had a further development in the process. And in 1970s, Stereovision technique, a breakthrough in the 3D development history came out, and was used in the movie field. (Two cameras were used in shooting movie, simulating human eyes, and then the movie was displayed with a projector projecting through a polarizing filter onto a movie screen, the audience watched the movie wearing polarized glasses.) In 1986, Canada developed the first 3D movie "Echos of the Sun" using polarized glasses. Gradually, 3D movie became a fashion, and 3D technology increasingly integrated into people's lives.

After television technology appears, people began to develop three-dimensional television. Conventional stereoscopic display methods used in film production were applied almost all to the stereo TV technology. With the development of Color television technology and Complementary color stereoscopic phase TV technology, Time-division stereoscopic television technology began to be gradually used in stereoscopic television.

When it comes to 21st century, a new generation of LCD and plasma display apparatus took the market, represented by its exquisite image quality, full-color, energy saving, no radiation, and no flicker. With the rapid development of display devices, people begin to focus on two directions, the three-dimensional display of the naked eye direction and the immersive 3D direction.

With the astonishing development of hi-tech, the functions and service of digital products are improved constantly. In that situation, game consoles need become more intelligent to provide customers a better play experience, such as more portable and slight appearance, better 3D effect. In this context, we designed a new product based on the new technologies to meet nowadays users' demands which we called it Immerse Helmet. 


\section{A NEW GAME CONSOLE WITH HI-TECH}

Immerse Helmet is a portable handheld game console which enables 3D display when user plays games or watches movies with it. Different from other 3D display equipment, Immerse Helmet is not just a 3D display monitor, in fact, it is an all-in-one game play station which can work independently without any outer equipment. The new chips of processing unit, OLED display and improved 3D display technology enable Immerse Helmet to have a faster processing capacity, and more real 3D feeling as well.

\subsection{New Chips of Processing Unit}

The typical game consoles have two modes - CPU and GPU separately or APU to implement the functions. CPU implements computing while GPU implements graphic processing (especially for $3 \mathrm{D}$ graphics). APU is to integrate CPU and GPU to one chip by engine fusion. APU integrates the CPU's function of doing precise scalar operations and the GPU's function of doing massive vector operations.

It is illustrated in the simplified model of CPU\&GPU and APU (Figure 1) that these components provide both computing and graphic processing capabilities. Because of the nonintegrated architectural feature of separate CPU and GPU, it has a larger volume and weight. The current APU has the defect of imbalance capability of CPU and GPU, which means that GPU is not powerful enough compared with CPU, especially in 3D graphic processing.
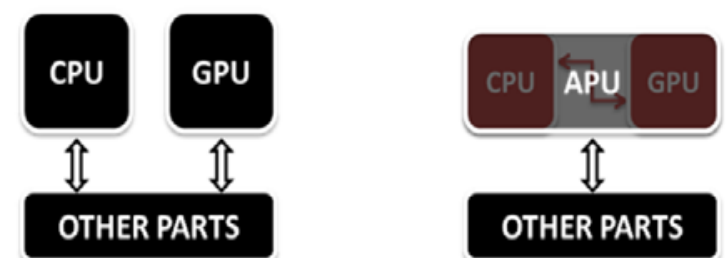

Figure 1. The simplified model of CPU\& GPU (left) and APU (right)

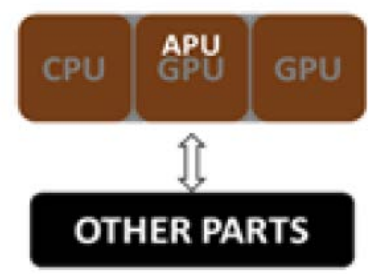

Figure 2. New APU

Today's general purpose GPU (GPGPU) computing offers unprecedented performance by offloading computing-intensive portions of the application to the GPGPU, while running the remainder of the code on the central processing unit (CPU). A chip of updated APU (Figure 2), which integrate one CPU and two GPUs will be used. The way of integration is not to simply vertically or horizontally connect the processing units, but to optimize the platform and redesign the architecture to minimize the volume. The new form of chip will be a breakthrough for the game console to achieve coordinate memory of CPU and GPUs in order to enhance the speed of both computing and graphic processing.

\subsection{OLED Screen Technology}

LCD - Liquid Crystal Diode is a material interposed between liquid and solid, which needs background light to implement the function of giving out light. These features make LCD of high energy consumption and large space to build them, which hinder the volume minimization. Furthermore, LCD has the drawback of background light leakage, and the viewing angle is severely limited because the light are directed by the inner components, which is not ideal for the headmounted 3D display. 


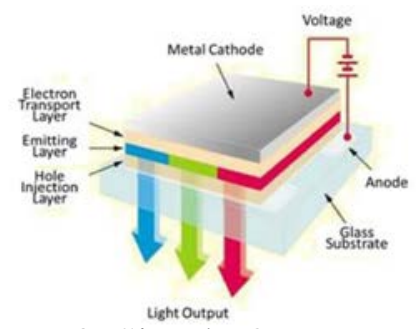

Figure 3. Simple OLED Model

To fit the 3D display, the technology of OLED (Organic Light Emitting Display) is used. OLED have shown its excellent performance on the new illumination and flat-panel display technology. A display screen can be divided into many tiny points. Voltage signal at each point lightens the layer of flo rescent material, and produce whether red, green or blue light.

As the simplified OLED shows (Figure 3), one component sends a work order, then the emitting layer produce a beam of red, green or blue light without direct it, finally the beams from each point mix together to implement colorful display function. OLED is suitable for small-size precise display.

\subsection{Immersive 3D Technology}

The traditional 3D, largely relies on red-and-green glasses, polarized glasses or shutter glasses, it is the non-immersive 3D technology. This kind of technology makes users experience the 3D view by adding a distance degree as shown in figure 4. However, users can only have a view of three dimension in a fixed direction from their eyesight, which means that the side of the scene cannot be seen. To break the width limitation of the scene, we choose the new immersive 3D technology to take the place.

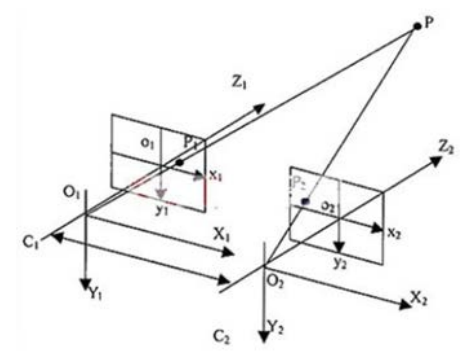

Figure 4. Non-Immersive 3D

There are several ways to realize immersive 3D experience, such as full-surround immersive design and eye tracking design. As a portable game console, we choose to use eye tracking as the main design, for that some other technologies need too much space, which is antithetical to the concept of the new product.

Eye tracking is currently being used in many fields, such as health and commercial studies. The process consists of measuring either the focus of attention (gaze) or eye movement in relation to the head.

At the present stage, there are 4 main methods to measure eye movement:

(1) EOG method. It uses electrodes to measure the voltage difference between the skins around the eye socket to realize the measurement of eye movements, and can identify three degrees of horizontal rotation and the eye 5 degrees vertical rotation.

(2) Scleral contact lens / search coil method, one of the most precise method to measure the eye movement, determines the orientation of the eye by measuring the azimuth of the attachments due to the rotation of the eye.

(3) POG, VOG method gets the movement information by measuring characteristics of the eye that can be distinguished.(Appearance of the shape of the pupil, iris and sclera Leis edge, Corneal reflex light sources near the eyes, etc.) 
(4) Video-based combination of the pupil and corneal reflection method, based on the coordinates of the corneal reflection and the pupil center point position coordinate, it can estimate line of sight vector.

Considering the properties of games, EOG method will easily produce fatigue, which is not suitable for long-time use. Scleral contact lens / search coil method, though outstanding in accuracy, is the most invasive way, also not apposite. The video-based combination of the pupil and corneal reflection method, which is both non-invasive and accurate, is our best choice.

Eye tracking technology will be used in the scene transitions in the game. Using the tracking technology, combined with the APU processing capability, the transition of scenes in the game's virtual world can be much closer to that in the real living world, providing increasing immersion to users.

The new immersive 3D technology provides not only the distance degree, but also width degree to construct a xyz-axis 3D view. With eye tracking technology, the users may have a view in any direction. Combined with the help of position control by the handle, the users are allowed to move in the view to see every corner or even go inside to see the inner structure without distortion. This technology perfectly restores the real scene and the scene is theoretically infinite.

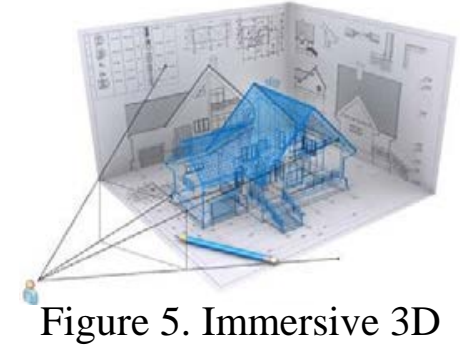

\section{DISCUSSION}

Our new product, employing new chips of processing units, OLED screen technology, and new immersive technology, provides users a better experience. But meanwhile, there are still some problems waiting to be solved.

The new chip is highly integrated to limit the relative volume. Double GPU improves the power of graphic processing and overcomes the imbalance of processing capability. Also the chip is lowpower to save energy. However, multithreading in multicore system architecture introduces challenges, such as data parallelism and synchronization, and the special motherboard of this chip is of high cost than the ordinary one.

OLED has the feature of self-emitting, which enables the game console to abandon the large background light part to reduce thickness and weightiness, and require less energy power as well. Besides, since the direction of light is not limited, it has a great viewing angle, which fits the demand of 3D display. However, OLED also has a disadvantage that it has a relatively short life. Scientists are doing efforts to overcome this shortage.

The immersive 3D technology has the superiority of 3-dimensional scenes restoration, building a 360-degree environment, which guarantees a better user experience of 3D. Furthermore, this will be a revolutionary update of portable game console to improve the degree of user immersion. However, this technology has some disadvantages. The first one is that the components need more volume than traditional 3D technology, but the volume increment can be offset by the size minimization of other components. The second one is the sense of discomfort, wearing the immersive 3D device too long may bring fatigue to the users, making the eyes jaded.

\section{CONCLUSION}

With the development of computer game devices and technologies, we think the combination of hi-tech is an inexorable trend. The Immerse Helmet just cater the trend, we believe that this device will lead the game equipment into a new wearable device domain. It will arouse the interest and attention of many developers in different research fields, and we are looking forward to the 
possibility of virtual reality technology to be used to develop this Immerse Helmet.

\section{References}

[1] Almeida, S. 2011. The eyes and games: A survey of visual attention and eye tracking input in video games [C]. In Proc. SBGames: X Brazilian Symp. Comput. Games Dig. Entertainment Arts Design Track: 1-10.

[2] Andrews, J. et al. 2006. Xbox 360 system architecture [J]. Micro, IEEE. 26(2): 25-37.

[3] Asaduzzaman, A. et al. 2014. GPU Computing to Improve Game Engine Performance [J]. Journal of Engineering and Technological Sciences, 46(2): 226-243.

[4] Björk, S. et al. 2002. Designing ubiquitous computing games-a report from a workshop exploring ubiquitous computing entertainment [J]. Personal and Ubiquitous Computing, 6(56): 443-458.

[5] Bian Fen.et al. 2009. Gaze tracking technology and its application [J]. Ergonomics, 15(1):48-52.

[6] Chen, William. 2001.Picture video signal converting/processing circuit for GBA (gameboy advance) or GBC (gameboy color).U.S. Patent Application 09/948,559 [P].

[7] Chung, B. Y., \& Kwon, O. K. 2013. U.S. Patent No. 8,593,378. Washington, DC: U.S. Patent and Trademark Office [P].

[8] Duchowski, A.et al. 2000. Eye-based interaction in graphical systems: Theory and practice [J]. ACM SIGGRAPH 2000 Course Notes:5.

[9] Daim, T. et al. 2014. Identifying and forecasting the reverse salient in video game consoles: A performance gap ratio comparative analysis [J]. Technological Forecasting and Social Change, 82: 177-189.

[10] Gather, M. et al. 2011. White Organic Light-Emitting Diodes [J]. Advanced Materials, 23(2): 233-248.

[11] Graetz, J. M. 1981. The origin of Spacewar [J]. Creative Computing: 18.

[12] Iizuka, K. 2006. Welcome to the wonderful world of 3D: introduction, principles and history [J]. Optics and Photonics News, 17(7):42-51.

[13] Kuchera-Morin, J. A. et al. 2014. Immersive full-surround multi-user system design [J]. Computers \& Graphics, 40: 10-21.

[14] Laird, J. E. 2005. History of Computer Games [J]. EECS Department, 9(7): 05.

[15] Lee, J. C. 2008. Hacking the nintendo wii remote [J]. Pervasive Computing, IEEE, 7(3): 39-45.

[16] Schilling, M. A. 2003. Technological leapfrogging: Lessons from the US videogame industry [J]. Available at SSRN 2533203.

[17] Tang, M. et al. 2015. Localized surface plasmons enhanced color conversion efficiency in organic light-emitting device with surface color conversion layer [J]. Synthetic Metals, 199: 69-73. 